\title{
Quantification of 23 Volatile Organic Compounds with a Single Reference Material Using Post-column Reaction Gas Chromatography Combined with a Stainless-steel Heating Furnace
}

\author{
Yuko Kitamaki, ${ }^{* \dagger}$ Naoki SaIto, ${ }^{*}$ Nanako SaSAKI,** Mariko Morita,** Tomohiro SaSAKI,** \\ Hideaki MiYamoto, ${ }^{* *}$ Masahiko Numata, ${ }^{*}$ and Toshihide IHARA* \\ *National Metrology Institute of Japan (NMIJ), National Institute of Advanced Industrial Science and \\ Technology (AIST), 1-1-1 Umezono, Tsukuba, Ibaraki 305-8563, Japan \\ **Horiba Stec Co., Ltd., 11-5 Hokotate Kamitoba, Minami, Kyoto 601-8116, Japan
}

\begin{abstract}
We built a heating furnace using stainless-steel instead of aluminum in gas chromatography combined with an oxidation/ reduction system; it increased the oxidation temperature to $650^{\circ} \mathrm{C}$. At $600^{\circ} \mathrm{C}$, it completely oxidized five organochlorine compounds. This system was applied to a standard solution of 23 volatile organic compounds. The analytical results of 20 hydrocarbon and organochlorine compounds showed good agreement with the expanded uncertainty $(k=2)$ of the reference values. Three organobromine compounds obtained values higher than the reference; this was investigated further.
\end{abstract}

Keywords Post-column reaction gas chromatography, volatile organic compound, organochlorine compounds, organobromine compounds

(Received November 18, 2020; Accepted February 1, 2021; Advance Publication Released Online by J-STAGE February 5, 2021)

\section{Introduction}

The preparation of the calibration curve for each target analyte is essential for accurate quantification with chromatographic techniques because the detector responses are strongly dependent on both analytes and the analytical conditions. However, reliable pure materials or standard solutions are not always available for all of the target analytes, and the preparation of a reliable standard solution containing many analytes is complicated and troublesome. Thus, a calibration curve commonly applicable to various analytes is required.

Flame ionization detectors (FIDs) have been widely used as universal detectors combined with gas chromatography (GC), and the FID responses are affected by other atoms and bonding types in the analytes' structures. ${ }^{1}$ Recently, a post-column reaction system, which converts the analytes' carbons to methane via carbon dioxide $\left(\mathrm{CO}_{2}\right)$ by using oxidation and reduction catalysts (the settable maximum reaction temperature is $400^{\circ} \mathrm{C}$ at the conventional system), followed by the detection of methane with FID, was developed and used with gas chromatography (post-column reaction GC). ${ }^{2-6}$ Because this system can detect all of the carbons in any compound such as methane, ideally, its FID responses more strictly reflect the number of carbons than conventional FID ones. Thus, this technique is emerging as a reliable and convenient strategy for quantifying various types of organic compounds containing not only carbon, hydrogen, and oxygen, but also nitrogen and sulfur. $^{7,8}$ In contrast, although application notes have been provided by a manufacture, ${ }^{9}$ there are no studies sufficiently on

† To whom correspondence should be addressed.

E-mail: y-kitamaki@aist.go.jp the application of this technique to organohalogen compounds, such as organochlorine and -bromine compounds; this should be partly attributed to their relatively poor oxidation characteristics, suspected from the polychlorinated biphenyls and brominated flame retardants.

In Japan, the Ministry of Health, Labor and Welfare has established drinking water quality standards in accordance with the World Health Organization (WHO) guidelines, listing more than 10 targets of volatile organic compounds (VOCs) and covering both hydrocarbons and organohalogen compounds. ${ }^{10}$ Thus, a standard solution containing 23 VOCs can be used for examining the applicability to the simultaneous quantification of organohalogen compounds with a single reference material. In this study, we built a heating furnace with stainless-steel to increase the oxidation temperature to $650^{\circ} \mathrm{C}$. The oxidation achievement of five organochlorine compounds was examined at 400,500 , and $600^{\circ} \mathrm{C}$ without reduction (in the oxidation mode). Because their complete oxidation was achieved at $600^{\circ} \mathrm{C}$, we applied this condition to a commercially available standard solution containing 23 VOCs. We also examined why organobromine compounds exhibited analytical results higher than the reference values.

\section{Experimental}

\section{Chemicals and preparation of testing mixture}

NMIJ CRMs of five high-purity volatile organochlorine compounds, dichloromethane (NMIJ CRM 4005-a), chloroform (NMIJ CRM 4007-a), carbon tetrachloride (NMIJ CRM 4006-a), 1,1,2-trichloroethane (NMIJ CRM 4016-a), and tetrachloroethylene (NMIJ CRM 4008-a) were obtained from National Metrology Institute of Japan (NMIJ). A NMIJ CRM of a high-purity 
hydrocarbon, toluene (NMIJ CRM 4003-a), was also obtained from NMIJ. Dibromochloromethane (reagent grade), tribromomethane (reagent grade), and methanol (infinity pure grade) were obtained from FujiFilm Wako Pure Chemical Corporation (Osaka, Japan). A standard solution containing 23 VOCs (each $1000 \mathrm{mg} \mathrm{L}^{-1}$ in methanol, JCSS grade) was obtained from Kanto Chemical Co. (Tokyo, Japan). The Japan Calibration Service System (JCSS) is that the metrological traceability system under the Measurement Act in Japan. ${ }^{11}$ Concentrations of 23 VOCs in the standard solution were calibrated by gas chromatography with measurement standards traceable to specified reference materials (national measurement standards). Bromodichloromethane (reagent grade) was obtained from AccuStandard Inc. (New Haven, CT, USA).

Prior to use, the purity of the organobromine compounds (bromodichloromethane, dibromochloromethane, and tribromomethane) was evaluated using the area normalization method of GC-FID and the stabilizer amounts. Here, dibromochloromethane and tribromomethane contained ethanol as a stabilizer. Therefore, each ethanol was quantified by a calibration curve method using the ethanol standard, and each purity by the area normalization method was corrected.

A test mixture (Mixture A) containing toluene, dichloromethane, chloroform, carbon tetrachloride, 1,1,2-trichloroethane, and tetrachloroethylene was prepared by mixing and diluting with methanol at $\sim 2460 \mathrm{mg} \mathrm{kg}^{-1}$ for each compound in $12 \mathrm{~mL}$ screw vials capped with Mininert valves (GL Sciences Inc. Tokyo, Japan). Another test mixture (Mixture B) containing toluene, bromodichloromethane, dibromochloromethane, and tribromomethane was prepared in the same manner as Mixture A, but at $\sim 1260 \mathrm{mg} \mathrm{kg}^{-1}$ for each compound.

\section{Instruments and analytical conditions}

The post-column reaction GC used was a combination of Agilent 7890A GC-FID (Agilent Technologies Inc., Santa Clara, CA, USA) and a Standard Materials Calibration System TE1000 (Horiba Stec Co., Ltd., Kyoto, Japan). Next, to ensure the complete oxidation of organochlorine compounds, some aluminum components of the heating furnace were replaced with stainless-steel components (SUS 430), allowing the system's use at temperatures up to $650^{\circ} \mathrm{C}$. A mass flow controller, SEC-Z512MGX (Horiba Stec Co., Ltd.), was used to keep the carrier gas flow rate constant. The operating conditions of the post-column reaction GC are summarized in Table S1 (Supporting Information).

\section{Quantification of target analytes using toluene as reference material}

The concentration of each analyte was calculated using Eq. (1), and toluene was used as the reference material (r):

$$
C_{\text {ana }}=\frac{A_{\text {ana }}}{A_{\mathrm{r}}} \times \frac{M_{\text {ana }}}{M_{\mathrm{r}}} \times \frac{N_{\mathrm{r}}}{N_{\text {ana }}} \times C_{\mathrm{r}},
$$

where $C_{\text {ana }}$ denotes the mass concentration of each analyte in the sample solution $\left(\mathrm{mg} \mathrm{kg}^{-1}\right) ; A_{\text {ana }}$, peak area of each analyte in the sample solution; $A_{\mathrm{r}}$, peak area of the reference material in the sample solution; $M_{\text {ana }}$, molar mass of each analyte $\left(\mathrm{g} \mathrm{mol}^{-1}\right) ; M_{\mathrm{r}}$, molar mass of the reference material $\left(\mathrm{g} \mathrm{mol}^{-1}\right) ; N_{\text {ana }}$, number of carbon atoms in each analyte; $N_{\mathrm{r}}$, number of carbon atoms in the reference material; and $C_{\mathrm{r}}$, mass concentration of the reference material in the sample solution $\left(\mathrm{mg} \mathrm{kg}^{-1}\right)$. Here, the molar masses were calculated using data from the IUPAC Table of Atomic Weights of the Elements, 2016. ${ }^{12}$
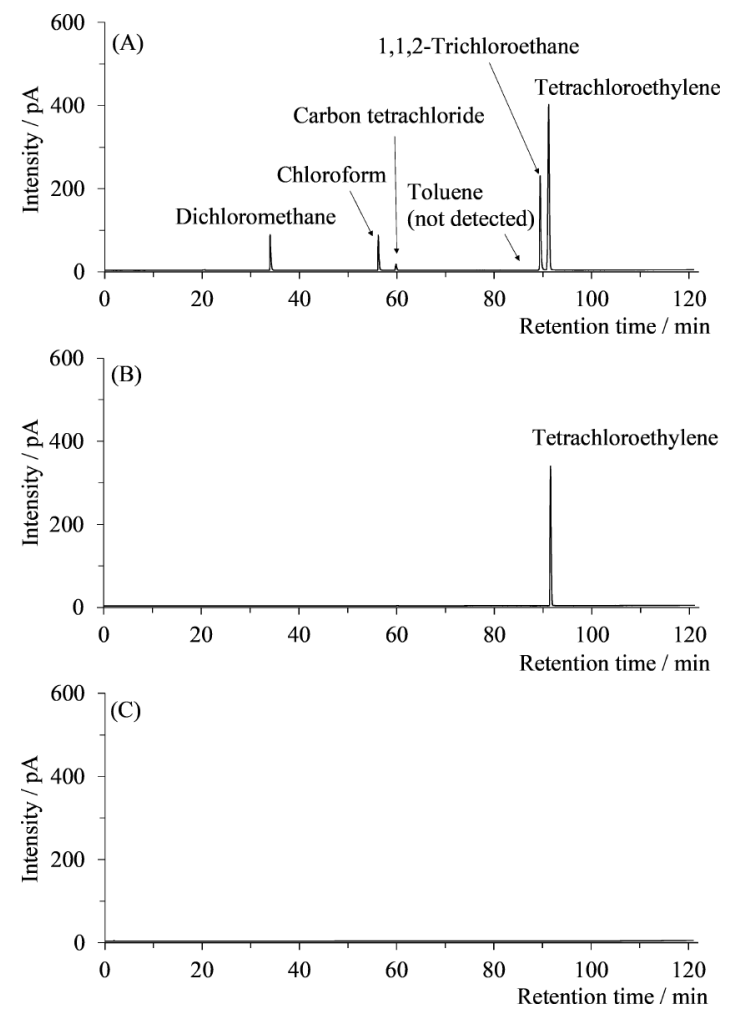

Fig. 1 Post-column reaction GC chromatograms of the test mixture (Mixture A) in the oxidation mode at different oxidation reaction temperatures. (A) Catalyst temperature, $400^{\circ} \mathrm{C}$; (B) catalyst temperature, $500^{\circ} \mathrm{C}$; (C) catalyst temperature, $600^{\circ} \mathrm{C}$.

\section{Results and Discussion}

Oxidation achievement depending on temperature at 400, 500, and $600^{\circ} \mathrm{C}$

A relatively high temperature enhances the oxidation process in theory; thus, an increase in the temperature of the heating furnace is expected to achieve a higher oxidation efficiency for organochlorine compounds. Here, $\mathrm{CO}_{2}$ produced by oxidizing organic compounds is undetectable with FID. Thus, we examined the oxidation achievement of the target compounds by detecting $\mathrm{CO}_{2}$ with FID (without the reduction process: oxidation mode).

Figure 1 shows FID chromatograms of Mixture A containing toluene and five organochlorine compounds at 400, 500, and $600^{\circ} \mathrm{C}$ in the oxidation mode. At $400^{\circ} \mathrm{C}$, the settable maximum temperature of the oxidation furnace with the conventional system, all of the peaks of the organochlorine compounds were observed, but a peak of toluene was not observed. This result suggested that this temperature was not sufficient to oxidize organochlorine compounds. At $500^{\circ} \mathrm{C}$, a large peak of tetrachloroethylene was still observed, but the peaks of the other organochlorine compounds disappeared. Finally, at $600^{\circ} \mathrm{C}$, all of the peaks of the organochlorine compounds in Mixture A completely disappeared. This result suggested in that all of the organochlorine compounds could be completely oxidized at $600^{\circ} \mathrm{C}$.

Application and validation with standard solution containing 23 VOCs

The heating-furnace-at $-600^{\circ} \mathrm{C}$ system was applied to the standard solution containing 23 VOCs including organochlorine 
Table 1 Compounds and their reference values in the standard solution with corresponding analytical results

\begin{tabular}{|c|c|c|c|c|c|c|}
\hline \multirow{2}{*}{$\begin{array}{l}\text { Peak } \\
\text { No. }\end{array}$} & \multirow{2}{*}{ Compound } & \multicolumn{2}{|c|}{ Reference values ${ }^{\mathrm{a}} / \mathrm{mg} \mathrm{L}^{-1}$} & \multicolumn{2}{|c|}{ Analytical results/mg L $\mathrm{m}^{-1}$} & \multirow{2}{*}{$\begin{array}{c}\text { Difference, } \\
\%\end{array}$} \\
\hline & & Concentration & $U^{\mathrm{b}}$ & Concentration & $\mathrm{SD}^{\mathrm{d}}$ & \\
\hline 1 & 1,1-Dichloroethylene & 1018 & 17 & 1000 & 5 & -1.8 \\
\hline 2 & Dichloromethane & 1006 & 11 & 1003 & 10 & -0.3 \\
\hline 3 & trans-1,2-Dichloroethylene & 1007 & 13 & 1002 & 2 & -0.5 \\
\hline 4 & cis-1,2-Dichloroethylene & 1005 & 10 & 1002 & 3 & -0.3 \\
\hline 5 & Chloroform & 1010 & 8 & 1015 & 4 & 0.5 \\
\hline 6 & 1,1,1-Trichloroethane & 1028 & 9 & 1029 & 9 & 0.1 \\
\hline 7 & Carbon tetrachloride & 1008 & 9 & 1005 & 15 & -0.3 \\
\hline 8 & Benzene & 1007 & 9 & 996 & 7 & -1.1 \\
\hline 9 & 1,2-Dichloroethane & 1003 & 8 & 981 & 13 & -2.2 \\
\hline 10 & Trichloroethylene & 1006 & 8 & 1002 & 3 & -0.4 \\
\hline 11 & 1,2-Dichloropropane & 1000 & 7 & 1000 & 4 & 0.0 \\
\hline 12 & Bromodichloromethane & 1002 & 8 & 1101 & 7 & 9.9 \\
\hline 13 & cis-1,3-Dichloropropene & 1009 & 34 & 994 & 5 & -1.5 \\
\hline 14 & Toluene (Reference) & 1004 & 8 & 1004 & - & - \\
\hline 15 & trans-1,3-Dichloropropene & 1012 & 47 & 995 & 2 & -1.7 \\
\hline 16 & 1,1,2-Trichloroethane & 1002 & 11 & 1020 & 2 & 1.8 \\
\hline 17 & Tetrachloroethylene & 1005 & 9 & 999 & 7 & -0.6 \\
\hline 18 & Dibromochloromethane & 1005 & 8 & 1168 & 10 & 16.2 \\
\hline $19+20^{c}$ & $p$-Xylene $+m$-xylene & 2010 & 15 & 2001 & 5 & -0.4 \\
\hline 21 & $o$-Xylene & 1006 & 7 & 1003 & 3 & -0.3 \\
\hline 22 & Tribromomethane & 1019 & 10 & 1230 & 20 & 20.7 \\
\hline 23 & 1,4-Dichlorobenzene & 1005 & 8 & 998 & 3 & -0.7 \\
\hline
\end{tabular}

a. Reference values of 23 VOCs are values shown on the certificate. b. $U$, expanded uncertainty $(k=2)$. c. The total reference value of the $p$-xylene and $m$-xylene $\left(2010 \mathrm{mg} \mathrm{L}^{-1}\right)$ was calculated from reference values of $p$-xylene $\left(1005 \mathrm{mg} \mathrm{L}^{-1}\right)$ and $m$-xylene $\left(1005 \mathrm{mg} \mathrm{L}^{-1}\right)$, and its expanded uncertainty $(k=2)$ was calculated from the square root of the sum of the squares of each expanded uncertainty $\left(11 \mathrm{mg} \mathrm{L}^{-1}\right.$ for $p$-xylene and $10 \mathrm{mg} \mathrm{L}^{-1}$ for $m$-xylene). d. Repeatability in SD was obtained from quintuplicated measurements $(n=4)$.

and -bromine compounds, and the analytical results were validated by comparing with the corresponding reference values.

Table 1 summarizes the reference values and the analytical results for the 23 VOCs examined, and the FID chromatogram of the 23 VOCs in the standard solution is shown in Fig. 2. Under our analytical condition, the 23 VOCs could be completely separated in over $200 \mathrm{~min}$, except for $p$-xylene and $m$-xylene. We used toluene as a reference compound for quantifying other VOCs, because it is constituted of carbon and hydrogen and eluted 14 of the 23 compounds examined. For all of the 23 VOCs, the analytical results showed good repeatability $(<3 \%$ in RSD, $n=4)$; they were within the range of the expanded uncertainty for all of the compounds, except for three organobromine compounds. This fact suggested that complete oxidation could be achieved, at least, for compounds constituted of both hydrogen and carbon and chlorine, by using the heating furnace at $600^{\circ} \mathrm{C}$; thus, the analytical results were comparable to the corresponding reference values. Therefore, a post-column reaction GC combined with a stainless-steel heating furnace at $600^{\circ} \mathrm{C}$ could be used for accurately quantifying hydrocarbon and organochlorine compounds with a single reference material within the $<3 \%$ range. However, three organobromine compounds were out of the expanded uncertainty $(k=2)$, and their analytical results were $\sim 10-21 \%$ higher than the reference values.

\section{Factor causing higher values for organobromine compounds}

Because incomplete oxidation introduces lower analytical values, there should be another factor responsible for the higher analytical results of the organobromine compounds. Here, we suspected an unexpected reaction of a bromine-substituted group with something in the system, because this phenomenon was observed only for organobromine compounds, and

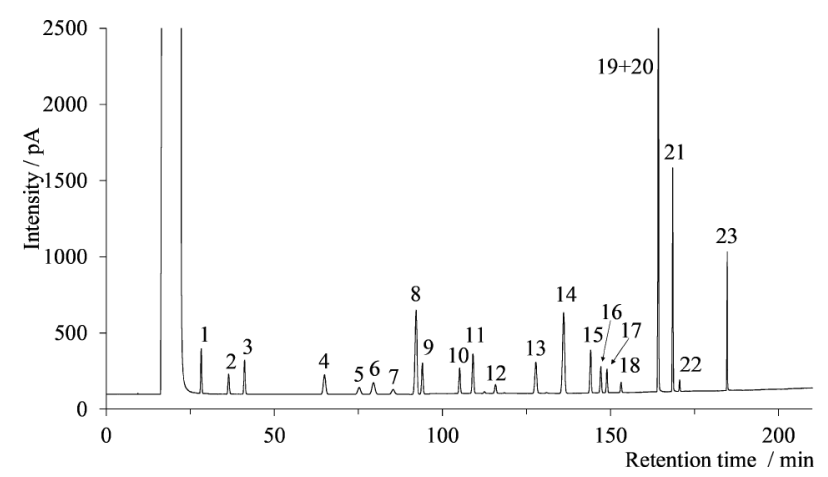

Fig. 2 Post-column reaction GC chromatogram of the 23 volatile organic compounds in the standard solution. The peak numbers in this figure correspond to those in Table 1.

tribromomethane $\left(\mathrm{CHBr}_{3}\right)$ showed a larger difference than both bromodichloromethane $\left(\mathrm{CHCl}_{2} \mathrm{Br}\right)$ and dibromochloromethane $\left(\mathrm{CHClBr}_{2}\right)$.

We found some reaction schemes for brominated compounds and cyano-groups with palladium catalysts in the previous studies, ${ }^{13,14}$ and the stationary phase of the BPX-VOLATILES column used for the 23-VOC separation was cyanopropylphenyl siloxane. Thus, we suspected that the reaction of organobromine compounds with cyanopropylphenyl siloxane was responsible for the higher analytical results. To examine this hypothesis, we prepared a mixture containing toluene, bromodichloromethane, dibromochloromethane and tribromomethane (Mixture B) by gravimetric blending, and analyzed this solution with a DB-5 column (stationary phase: (5\% phenyl)-methylpolysiloxane), although this column had relatively poor separation properties 
Table 2 Preparation values and analytical results of three organobromine compounds examined (Mixture B)

\begin{tabular}{|c|c|c|c|c|c|}
\hline \multirow{2}{*}{ Compound } & \multicolumn{2}{|c|}{ Preparation value $/ \mathrm{mg} \mathrm{kg}^{-1}$} & \multicolumn{2}{|c|}{ Analytical results $/ \mathrm{mg} \mathrm{kg}^{-1}$} & \multirow{2}{*}{$\begin{array}{c}\text { Difference, } \\
\%\end{array}$} \\
\hline & Concentration & $U^{\mathrm{b}}$ & Concentration & $\mathrm{SD}^{\mathrm{c}}$ & \\
\hline Bromodichloromethane & 1256 & 23 & 1283 & 11 & 2.2 \\
\hline Dibromochloromethane & 1253 & 60 & 1270 & 18 & 1.4 \\
\hline Tribromomethane & 1261 & 22 & 1255 & 9 & -0.5 \\
\hline
\end{tabular}

a. Preparation values of bromodichloromethane, dibromochloromethane and tribromomethane were decided by the gravimetric blending. b. $U$, expanded uncertainty $(k=2)$. The expanded uncertainty $(k=2)$ of the preparation value was estimated from uncertainties originated from the preparation $(0.2 \%)$ and each purity (bromodichloromethane $0.9 \%$, dibromochloromethane $2.3 \%$, and tribromomethane $0.9 \%$ ).

c. Repeatability in SD was obtained from quintuplicated measurements $(n=5)$.

of the 23 VOCs. Table 2 summarizes the preparation values and the analytical results. The analytical results of the three organobromine compounds examined were comparable to the preparation values with good repeatability ( $<2 \%$ in $\mathrm{RSD}, n=5)$; the differences between the analytical results and the preparation values clearly improved by $<3 \%$, and were thus comparable to those of other hydrocarbon and organochlorine compounds with a cyanopropylphenyl siloxane stationary phase (Table 1). Therefore, the higher analytical results obtained with a BPXVOLATILES column, which was the most suitable column for the separation of the $23 \mathrm{VOCs}$, could be attributed to the reaction of the organobromine compounds with the cyano-substituted group originating from the column bleed.

\section{Conclusions}

The heating furnace for oxidation was replaced with a stainlesssteel component, which enabled us to increase the temperature to $650^{\circ} \mathrm{C}$. At $600^{\circ} \mathrm{C}$, compounds constituted from not only hydrogen and carbon, but also chlorine, were completely oxidized, and their analytical results were within the expanded uncertainty range of the reference values in the $23 \mathrm{VOC}$ standard solution when toluene was used as the reference material. Although the organobromine compounds could also be oxidized at $600^{\circ} \mathrm{C}$, these compounds reacted with a cyano-substituted group originating from the column bleed of the stationary phase used for the separation, and introduced higher analytical results. Thus, the stationary phase having cyanopropyl-substituted groups should not be for avoiding the inaccurate quantification of organobromine components.

This method is expected to be applicable, for example, to characterization of the standard solution for tap water quality analysis. Nevertheless, further studies are necessary to apply this method to the quantification of VOCs containing other elements. Additional careful examinations are also required, for example, on the GC separation conditions and the matrix effects on the oxidation and reduction reactions, for investigating complex sample matrices, such as in environmental and food analyses.

\section{Supporting Information}

Detailed operating conditions of the post-column reaction GC are described in Supporting Information. This material is available free of charge on the web at http://www.jsac.or.jp/ analsci/.

\section{References}

1. M. Kállai, Z. Veres, and J. Balla, Chromatographia, 2001, $54,511$.

2. T. Watanabe, K. Kato, N. Matsumoto, and T. Maeda, Bunseki Kagaku, 2003, 4, 265.

3. C. A. Beach, C. Krumm, C. S. Spanjers, S. Maduskar, A. J. Jones, and P. J. Dauenhauer, Analyst, 2016, 141, 1627.

4. C. S. Spanjers, C. A. Beach, A. J. Jones, and P J. Dauenhauer, Anal. Methods, 2017, 9, 1928.

5. L. Bai, D. D. Carlton Jr., and K. A. Schug, J. Sep. Sci., 2018, 41, 4031.

6. Y. Kitamaki, N. Saito, M. Numata, and T. Ihara, Accredit. Qual. Assur., 2018, 23, 297.

7. T. Watanabe, T. Sasaki, T. Yoshimura, S. Narukami, and T. Shimosaka, Anal. Sci., 2018, 34, 853.

8. C. A. Beach, K. E. Joseph, P. J. Dauenhauer, C. C. Spanjers, A. J. Jones, and T. J. Mountziaris, American Institute of Chemical Engineers, 2017, 63, 5438.

9. Activated Research Company, Accurate Single Injection Quantification of Organochlorides Using the Polyarc ${ }^{\circledR}$ System (Activated Research Company (ARC), Minnesota, USA), http://www.activatedresearch.com/Public_Documents/ PA_Chlorinated\%20Compounds_1813.pdf.

10. Ministry of Health, Labor and Welfare, 4. Water quality, https://www.mhlw.go.jp/english/policy/health/water_ supply/4.html.

11. International Accreditation Japan, National Institute of Technology and Evaluation, Japan Calibration Service System (JCSS), https://www.nite.go.jp/en/iajapan/jcss/ index.html (Accessed 21 July 2020).

12. J. Meija, T. Coplen, M. Berglund, W. Brand, P. Bièvre, M. Gröning, N. Holden, J. Irrgeher, R. Loss, T. Walczyk, and T. Prohaska, Pure. Appl. Chem., 2016, 88, 265.

13. S. Zheng, C. Yu, and Z. Shen, Org. Lett., 2012, 14, 3644.

14. K. J. Powell, L. C. Han, P. Sharma, and J. E. Moses, Org. Lett., 2014, 16, 2158. 